\title{
Parametric Evaluation of Melting Practice on Induction Furnace to Improve Efficiency and System Productivity of CI and SGI Foundry- A Review
}

\author{
Mr. Digvijay D.Patil ${ }^{1}$, Prof. Dayanand A. Ghatge ${ }^{2}$ \\ Dept of Mechanical Engineering, Karmaveer Bhaurao Patil College of Engineering, Satara, Maharashtra, India ${ }^{1,2}$
}

\begin{abstract}
We provide some recommendations for the productivity improvement. Due to non-availability of the proper instrumentation the effect of the ill practices cannot be precisely judged. If this is properly measured, the percentage of productivity improvement in steel melting Induction Furnace can be calculated. The review is carried out from the literature in the various journals and manuals. The aim of this paper is to study the overall performance of induction furnace and to suggest the method to improve melt rate with optimum use of electricity. This paper mainly put attention on induction furnace as these are main consumer of electricity in foundry. In case of induction furnace efficiency is sensitive to many controllable features lie in operational practices, coil height; charge mix, furnace utilization etc. So with the help of recommendation, it is easy to find out the ways to lower the specific energy consumption in this furnace. So, in this paper we are trying to develop certain relationship between input and output parameters to improve the Whole process.
\end{abstract}

Keywords: Induction Furnace, Molten metal, Productivity, Melt rate, Energy Consumption.

\section{INTRODUCTION}

The development of Induction Furnaces starts as far back requirements for large castings, especially if off-shift as Michael Faraday, who discovered the principle of melting is practiced. It is widely used for duplexing electromagnetic induction. However it was not until the operations and installations where production late 1870's when De Ferranti, in Europe began requirements demand a safe cushion of readily available experiments on Induction furnaces. In 1890, Edward Allen molten metal. The coreless induction furnace is used when Colby patented an induction furnace for melting metals. a quick melt of one alloy is desirable, or it is necessary to The first practical usage was in Gysinnge, Sweden, by vary alloys frequently. The coreless furnace may be Kjellin in 1900 and was similar to the Colby furnace with completely emptied and restarted easily [1].

the primary closest to the core. The first steel made in an Induction furnaces have increased capacity to where induction furnace in the United States was in 1907 in a modern high-power-density induction furnaces are Colby furnace near Philadelphia.The first induction competing successfully with cupola melting (Fig.1).

furnace for three -phase application was built in Germany in 1906 by Rochling-Rodenhauser. The two basic designs of induction furnaces, the core type or channel furnace and the coreless, are certainly not new to the industry. The channel furnace is useful for small foundries with special

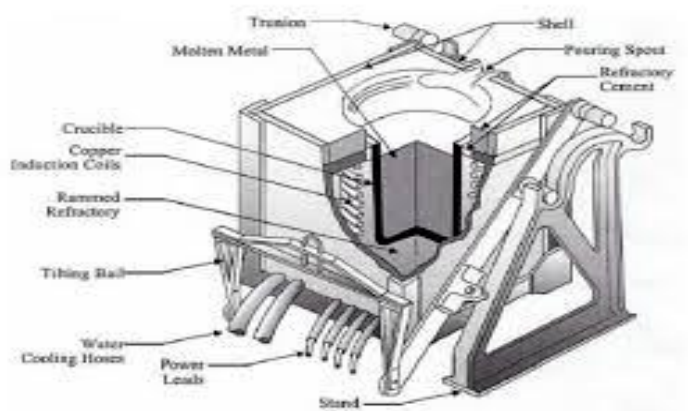

Fig.1: Schematic of induction furnace [1]

\section{METHODOLOGY STUDY OF INDUCTION FURNACE - A REVIEW}

The working of induction furnaces is based on the principle of electromagnetic induction and basic concept is same as that of a transformer but with a concept is same as that of a transformer but with, a single turn short circuited secondary winding. The charge to be heated and melted forms the secondary while the hollow water cooled copper coils excited by the A. C. supply from the primary. In the core type furnaces, in order to maintain the electric path there must always be a sufficient molten metal the furnace. This is called the molten heel. In the core less induction furnaces, the primary coils surround a refractory crucible in which the charge to be melted is put. The eddy currents induced by the primary winding generate heat in the charge. Since there is no core, a large current is required to 


\section{IAR JSET}

overcome the reluctance between the coils and the charge and results in a very low pf. The coil is surrounded by a laminated magnetic yoke to provide a return path for the flux to prevent stray losses and improve the pf. The whole furnace is contaminated in a mechanically rigid structure and mounted so that it can be tilted for pouring.[2]

\section{DOMESTIC STEEL SECTOR SCENARIO}

\section{A. Present Scenario:}

After 2 years of depressed market, the steel market has suddenly shown competitiveness. It is noted that induction-melting furnaces in various parts of the country are at present operating to near capacity. However, the power is not supplied to the units fully. Revolution is taking place to make steel in India by utilizing various technologies. therefore, emerging as a country with innovative idea to make steel, which is not followed by other countries in the world.

In the first decade of twenty first century, major existing integrated steel plants will face a challenge in producing Long products from Induction Furnaces in producing steel economically and efficiently. [2]

\section{B. Ferrous Scrap:}

The word "Ferrous" comes from the Latin word "Ferrum". Most people associate scrap with waste or rubbish. However, our Industry prefers to refer to ourselves as "Recyclers", who play a very important role, in not only feeding the Steel Industry but also protecting the environment by converting waste into wealth for society.

\section{Global Requirement for Scrap:}

With global steel production at 1 billion tonne mark, merchant scrap requirement is estimated in the current year at 318 million tonnes. By the year2010,requirement for merchant. scrap is likely to go up to 388 million tonnes. As the GDP grows in developing countries, the generation of merchant scrap will increase and additional processing capacities and scrap yards will have to be installed to meet the demand for quality scrap needed for the increasing steel demand[2].

\section{ELECTRIC INDUCTION FURNACE}

Induction furnace is an electrical furnace in which the heat is applied by induction heating of a conductive medium (usually a metal) in a crucible placed in a water-cooled alternating current solenoid coil [4]. Induction and arc furnaces use electricity to melt steel and cast iron. The advantage of the induction furnace is a clean, energyefficient and well-controllable melting process compared to most other means of metal melting. The electric induction furnace uses electric currents to melt metal. Induction furnaces are ideal for melting and alloying a wide variety of metals with minimum melt losses.

In the liquid steel is produced induction Furnace (IF) cast into ingots or continuously cast into blooms/billets/slabs for further rolling into desired product [4].

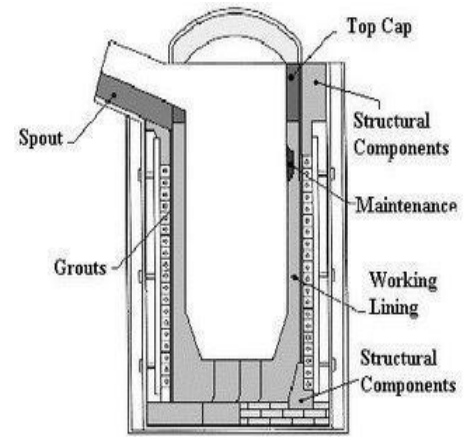

Fig. 2. Electric Induction Furnace [4]

The steel mills employing this process route are generally called as mini or midi steel plants. Since liquid steel after melting contains impurities like sulphur and phosphorus beyond desirable limits and no refining is generally possible in induction furnace. The structural steel produced through this process is inferior in quality. Quality can be further improved by secondary refining in the ladle furnace, vacuum degassing unit or vacuum arcdegassing (VAD) unit

\section{PRINCIPLE OF INDUCTION FURNACE}

The principle of induction furnace is the Induction heating. 1.Induction Heating: -Electromagnetic induction is a heating technique for electrical conductive materials(metals)[3]. Induction heating is frequently applied in several thermal processes such as the melting and the heating of metals. The heating speeds are extremely high because of the high power density. Induction heating is a form of non-contact heating for conductive materials. The principle of induction heating is mainly based on two well-known physical phenomena 1. Electromagnetic induction. 2.The Joule effect.

\section{A. Electromagnetic induction}

The energy transfer to the object to be heated occurs by means of electromagnetic induction. It is known that in a loop of conductive material an alternating current is induced, when this loop is placed in an alternating magnetic field $[3,5]$. When the loop is short-circuited, the induced voltage $\mathrm{E}$ will cause a current to flow that opposes its cause the alternating magnetic field. This is Faraday Lenz's law. The formula is $=/$; $\mathrm{U}=\operatorname{voltage}(\mathrm{V})$; = magnetic flux $(\mathrm{Wb}) ; \mathrm{t}=$ time $(\mathrm{s})$
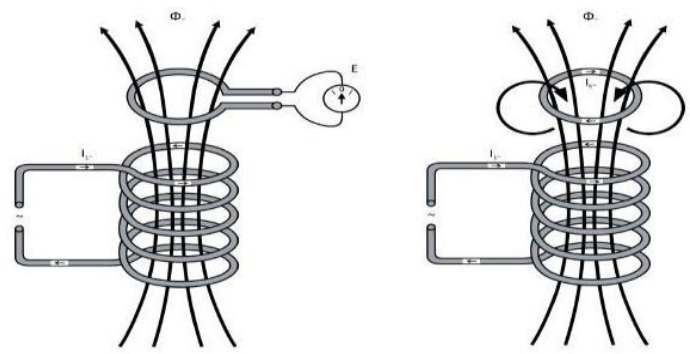

Fig. 3. Law of Electromagnetic Induction [3] 


\section{IAR JSET}

B. Joule's effect-

If a massive conductor (e.g. a cylinder) is placed in the alternating magnetic field instead of the sort circuited loop, then eddy current will be induced [3],[5]. The eddy current heat up the conductor according to the Joule effect. When a cur rent [A] flows through a conductor with resistance $\mathrm{R}[\mathrm{]}$, the power $\mathrm{P}[\mathrm{W}]$ is dissipated in the conductor. $=\times 2[$ ]

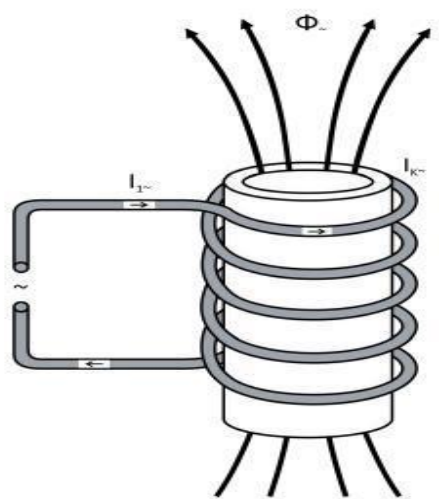

Fig. 4. Joule Effect or induction of Eddy currents [3]

\section{TYPES OF INDUCTION FURNACE}

There are two main types of induction furnaces:

1. Coreless Induction Furnace

2. Channel Induction Furnace

A. Coreless induction furnaces

The heart of the coreless induction furnace is the coil, which consists of a hollow section of heavy duty, high conductivity copper tubing which is wound into a helical coil [6]. Coil shape is contained within a steel shell. To protect it from overheating, the coil is water-cooled, the water being re-circulated and cooled in a cooling tower. The crucible is formed by ramming a granular refractory between the coil and a hollow internal. The coreless induction furnace is commonly used to melt all grades of steels and irons as well as many non-ferrous alloys. The furnace is ideal for re-melting and alloying because of the high degree of control over temperature and chemistry while the induction current provides good circulation of the melt

\section{B. Channel induction furnace}

The channel induction furnace consists of a refractory lined steel shell which contains the molten metal when it is attached to the steel shell and connected by a throat is an induction unit which forms the melting component of the furnace [6]. The induction unit consists of an iron core in the form of a ring around which a primary induction coil is wound. This assembly forms a simple transformer in which the molten metal loops comprises the secondary component The heat generated within the loop causes the metal to circulate Channel induction furnaces are commonly used for melting low melting point alloys and or as holding and superheating unit for higher melting point alloys such as cast iron. These furnaces basically consist of a vessel to which one or more inductors are attached.

\section{OBJECTIVES}

The aims and objectives of the present study are as follows:-

- To reduce production cost and improve System Productivity and Efficiency

- To reduce Power Consumption of Induction furnace.

- Providing better quality of products.

- Avoiding or reducing downtime and wastage of material.

- To identify the locations of bottlenecks and eliminate them

- To minimizing the heat losses

- To find opportunities to energy conservations

- Set a Standard procedure for melting on induction furnace.

\section{LITERATURE SURVEY}

The literature review reveals that a great deal of work has been carried out in the field of process improvement in the industries using Induction furnace for steel melting, It is observed that there is lot of work has been done on the parameters which are responsible to reduce the energy consumption and reducing the production cost of steel.

1) S. Jena at al. (1992) has studied the exposure to higher temperatures. Inductive power has been applied to the melting of high carbon ferrochromium fines at Zimasco [7]. They have focused on improving product quality, reducing the cost of production and new methods of casting the alloy separately from the slag. Investigations were carried out by them on the possibility of reducing the carbon content in the alloy. Attention was paid to increasing the number of heats obtained from each campaign by extending the life of the refractory lining. They have also devoted to optimizing the melting operation by automatic linkage of key process- control parameters.[7]

2) Pritibhushan Sinha at al. (1998) have made an optimum design of the lining of a medium frequency induction melting crucible frequently. They present a methodical approach for better design and maintenance to decrease such problems The erosion process of the lining is modelled by them as a renewal reward process. They have discussed the problems related to the erosion of the lining of an MFIM furnace, with a view to evolving practices which may improve the efficiency of such a furnace[8] 3) S.K. Dutta at al. (2004) have done studies on direct reduced iron melting in induction furnace. They have examined melting of DRI (direct reduced iron) in a laboratory size induction furnace using molten steel bath as hot heel [5].The effect of partial replacement of scrap 
IARJSET

by DRI on various melting parameters has been studied by them.[9]

4) Anuwat Pansuwan at al. (2009) have done work on temperature estimation of liquid steel in induction furnace. They presents The indirect measures of temperature by measuring cooling water Temperature at the outer wall lining of the induction furnace in order to estimate the temperature of the liquid steel in the furnace[10]. The technique for estimating the temperature relies on the consideration of the heat balance equation of the furnace and the use of several parameters for the furnace during the processes in order to estimateThe losses of heat from the furnace in order to calculate the heat balance equation. From this method, we can estimate the temperature of liquid steel in the induction furnace accurately[10]

5) Vivek R. Gandhewar et al. (2011) have done study on Induction Furnace. They have carried out pilot study in few industries in India, to verify the working practices and parameters of the Induction Furnaces [11]. In few cases they have observed lack of standardisation of process. Hence for improving its efficiency and for reducing the losses they have made recommendation like [11]

a) scheduling of operations

b) molten metal delivery

c) preheating, no time delay in holding the molten metal,

d) reuse of hot gases, using the good quality raw material,

e) proper charging practice.

6) E. Kardas (2012) have worked in evaluation of efficiency of working time of equipment in blast furnace. They have per-formed OEE and PAMCO analysis, which enabled to asses and identify factors which had great effect on efficiency of blast furnace department under study blast furnace department under study The level of efficiency is influenced by many factors Situation on the steel market in Poland and the world: changes in demand for steel products cause changes in the volume of production of pig iron, what effects on the efficiency of blast furnaces department. Demand for raw materials: changes in demand for raw materials on the market often cause situation when steel plants are forced to order raw materials which are low quality, what effects the quantity and quality of produced pig iron and quantity of slag Work organization of blast furnace - to maintain continuity of production and equipment in proper condition, company accepted the rule that while two blast furnaces work, the third undergoes renovation[12]

7) Sunil M. Jaralikar (2013) have studied the performance of an induction furnace in a steel melting industrial unit by employing a 12 pulse converter in its power supply unit [13]. Thus the furnace forms a nonlinear load and reflects all the negative effects associated with the inductive loads and power electronics based converter circuits. The performance analysis and harmonic measurements of the induction furnace under consideration with the existing setup of 12 pulse converter indicates that the specific consumption of the furnace is high when compared with the rated consumption, the distortions in the current and voltage waveform (THD) is beyond the permissible limits and that there is good scope for improvement in its performance and economy of operation. For improving the performance of this furnace, it is proposed to replace the existing 12 pulse converter with 24 pulse converter.

8) Sneha P. Gadpayle et al. (2014) have done study on electric melting furnace. The Induction furnace design and subsequently its fabrication should be promoted considering the abundant power sources, less maintenance cost and labour requirements [14]. It is observed by them that resistance that resistance heating is appropriate and energy efficient for a variety of heat treating, preheating processes in terms of cost of the furnace. As the furnace for melting of metals require different set ups due to the purpose of use.[14]

9) L.Smith has concluded that it is essential that consideration be given to energy costs both at purchase stage and throughout a furnaces useful life. It is through awareness of salient factors such as charge materials, charging practices, linings, temperature measurement and keeping that minimum energy costs can be attained. These costs should not be divorced from other factors such as recovery, flexibility and environment [15]

10) J.Powell has analysed in his paper presented at the institute of British foundries that in case of coreless furnaces, full power should be applied to the furnace for the maximum possible time to ensure maximum output and minimum energy consumption, and holding periods should be kept to a minimum. The use of medium frequency furnaces started from cold and emptied completely immediately after each melt can have better energy consumption figures, since there are few if any holding periods. In addition, the increased power densities available with such furnaces and their increased melting rate capability further reduce energy consumption. The type of refractory construction employed in channel furnaces can also affect energy consumption and lining wear has an adverse effect. With all furnaces, it is important to minimize heat losses by proper attention to linings and the use of lids and covers [15].

\section{DISCUSSIONS}

From the literature survey following points are needs to be discussed:-

- We have to focus on energy conservation, minimum consumption maximum output policy should be consumption maximum output policy should be

- Now-a-days demand of steel is increasing due to increase in infrastructure and globalization. And to meet this demand we are looking for such foundry process which will produce high quality steel with minimum time

- The improvement should be done in induction furnace and to improve melt rate with optimum use of electricity 


\section{IARJSET}

Vol. 4, Special Issue 1, January 2017

- Finite Element Method (FEM) approach may be used energy burden in another step. Molten metal where to reduce the design cost, time and efforts for any other additional energy is required to maintain the temperature required induction furnace The furnace was of the molten metal until it is poured. If a furnace can be constructed putting into consideration; its temperature scheduled so that it can operate at continuous full power, attainment, capacity of metals it can hold, the the energy requirements for melting steel can be reduced depth/surface area to be heat treated, operators safety, substantially.

space to be occupied in the workshop floor, cost While reviewing the literature, it is observed that till this restrictions, availability of the materials used, its time an attempt is not made by the concerns to study the maintainability and portability

- Furnace monitoring and billet cutting system may be adapted in order to increase the efficiency.

- Optimum thickness for reducing heat loss in furnace with economical cost is needed There are huge losses in the existing furnace hence we have concluded that optimization is necessary Conversion of waste to energy is one of the recent

- There are huge losses in the existing furnace hence we have concluded that optimization is necessary

- Conversion of waste to energy is one of the recent trends in minimizing not only the waste disposal tries.

- Latest techniques and machines should be used.

- The integration of the furnaces can be done to meet various requirements such as melting of metals. Various purposes can be achieved using the same

- Spillage of metal also incorporates the burning loss, as the burning loss is derived from loss of metal before pouring and after pouring, therefore it is very much necessary to reduce the spillage of metal while it is melted form.

\section{CONCLUSION}

Through the exhaustive review of literature, the basic operations of Induction Furnace and importance of its individual parameters are studied. Pilot study is out in few industries in India, to verify the working practices and parameters of the Induction Furnaces. It is found through observations that, there are many differences in operations in various industries. In few cases lack of standardization of process is also observed. In this paper we are focusing on improving the efficiency of steel melting processes. After actually watching all the steel melting process, we came to know that, what are the various losses and where heat is lost.

Hence for improving its efficiency and for reducing the losses we have made recommendation like Scheduling of operations, Molten metal delivery, Preheating, No time delay in holding the molten metal, Reuse of hot gases, Using The good quality raw material, Proper charging practice. If this comes in regular practice obviously it helps to increase its efficiency. Material and energy losses during these process steps represent inefficiencies that waste energy and increase the costs of melting operations. Modifying the design and/or operation of any step in the melting process may affect the subsequent steps. It is, therefore, important to examine the impact of all proposed modifications over the entire melting process to ensure that energy improvement in one step is not translating to impact of variation of one parameter on others. Through the planned design of experimentation, the effect of individual parameters can be studied and its overall effect on productivity can be found out

\section{REFERENCES}

[1] Vivek R. Gandhewar 2011, 277-284et al. / International Journal of Engineering and Technology Vol.3 (4),

[2] Ikbal Nathani (November 15, 2005) importance of recycling and current ferrous scrap scenario. Ambassador, Indian sub-continent bureau of international recycling Brussels

[3] Akash Khaitan, 26 th May-17th July, 2010 "A eport on Induction Furnace", Nikita Metals Kalyaneshwari, Burdwan, W.B. An Internship Program-II sta tion of Faculty of Science \& Technology, ICFAI University

[4] Prof. S. R. Satish Kumar and Prof. A. R. Santha Kumar, "De- sign of Steel Structures" Indian Institute of Technology Madras

[5] Jean Callebaut, Laborelec, 2014, "Application Note Induction Heating”, ECI Publication No Cu0123,

[6] Gulfam Hussain, "Electric Induction Furnace", http://www.slide share. net/e gulfam/induction-furnace-

[7] S. Jena and S.T. Ravastngadi, Johannesburg, SAIMM, 1992 , Pages 113-118 "Commissioning and Operating an Induction Furnace at Zimasco (KweKwe Division) to Melt High- carbon Ferrochromium", IFACON 6; Proceedings of the 6th International Ferroalloys Congress ,Cape Town, Volume 1,

[8] Pritibhushan Sinha and SubhashSaha 5, No. 4, Pages 255-259 Chandra, "An Optimum Design of the Lining of a Medium Frequency Induction Melting Furnace", International Transactions in Operational Research Vol

[9] S.K. Dutta, A.B. Lele and N.K. Pancholi, October 2004; Pages $467-$ 473 "Studies on Direct S.K. Dutta, A.B. Lele and N.K. Pancholi, "Studies on Direct Inst. Met.Vol.57, No. 5,

[10] Anuwat Pansuwan March 18 - 20, 2009, Hong Kong Temperature Estimation of Liquid Steel in Induction Furnace", Proceedings of the International Multi Conference of Engineers and Computer Scientists 2009 Vol II IMECS 2009

[11] Vivek R. Gandhewar, Satish V. Bansod, Atul B. Borade, Vol.3 (4), 2011, Pages 277-284 "In duction Furnace - A Review", International Journal of Engineer- ing and Technology

[12] E. Kardas, December 2012, Pages 876-880 "Evaluation of Efficiency of Working Time of Equip ment in Blast Furnace Department", Journal of Achievements in Materials and Manufacturing Engineering Volume 55 Issue 2

[13] Sunil M. Jaralikar, 7, July 2013, Pages3414-3421 "Performance Analysis of an Induction Fur nace Employing 12 Pulse ConverterA Case Study; International Journal of Advanced Research in Electrical", Electronics and Instrumentation Engineering Vol. 2, Issue

[14] Sneha P. Gadpayle, Rashmi N. Baxi ssue-5, March 2014, Pages 8083 "Electric Melting Furnace - A Review", International Journal of Emerging cience and En- gineering (IJESE) Volume-2,

[15] W.A Persons, J.S., L.smith (n.d.). Reduced output and short time working effect on energy consumption and costs for electric furnace operation's. 